\title{
Flow instability effects related to purge through a gas turbine chute seal
}

\section{Original article}

Article history:

Submission date: 23 November 2020

Acceptance date: 12 July 2021

Publication date: 24 August 2021

This is the updated version of a paper originally presented at the Global Power and Propulsion Virtual Technical Conference, GPPS Chania 20, Sept. 7-9, 2020.

\section{Check for updates}

*Correspondence:

ASR: arijit@kth.se

\section{Peer review:}

Single blind

\section{Copyright:}

(c) 2021 Roy et al. @ This is an open access article distributed under the Creative Commons Attribution License (CC-BY 4.0), which permits unrestricted use, distribution, and reproduction in any medium, provided the original work is properly cited and its authors credited.

\section{Keywords:}

purge flow; chute seal; axial turbine; rotational instabilities; ingress; flow coefficient

\section{Citation:}

Roy A. S., Fridh J., Scobie J. A., Sangan C. M., and Lock G. D. (2021). Flow instability effects related to purge through a gas turbine chute seal. Journal of the Global Power and Propulsion Society. 5: 111-125. https://doi.org/10.33737/jgpps/140172
Arijit Sinha Roy ${ }^{1, *}$, Jens Fridh ${ }^{1}$, James A. Scobie ${ }^{2}$, Carl M. Sangan $^{2}$, Gary D. Lock ${ }^{2}$

${ }^{1}$ KTH Royal Institute of Technology, Brinellvägen 8, SE-114 28, Stockholm, Sweden

${ }^{2}$ University of Bath, Claverton Down, Bath BA2 7AY, UK

\begin{abstract}
This paper investigates flow instabilities inside the cavity formed between the stator and rotor disks of a high-speed turbine rig. The cavity rim seal is of chute seal design. The influence of flow coefficient on the sealing effectiveness at constant purge flow rate through the wheel-space is determined. The effectiveness at different radial positions over a range of purge flow conditions and flow coefficients is also studied. Unsteady pressure measurements have identified the frequency of instabilities that form within the rim seal, phenomena which have been observed in other studies. Frequencies of these disturbances, and their correlation in the circumferential direction have determined the strength and speed of rotation of the instabilities within the cavity. Large scale unsteady rotational structures have been identified, which show similarity to previous studies. These disturbances have been found to be weakly dependent on the purge flow and flow coefficients, although an increased purge reduced both the intensity and speed of rotation of the instabilities. Additionally, certain uncorrelated disturbances have been found to be inconsistent (discontinuous) with pitchwise variation.
\end{abstract}

\section{Introduction}

Ingress of hot gases into the cavity or wheel space between the rotor and the stator is an important issue to the turbine designer (Dahlqvist, 2017). The gas at the turbine inlet has high kinetic and thermal energy and ingress is detrimental to the operational quality and durability of highly-stressed rotating components. Designers use various counter measures to prevent or minimize this phenomenon. Relatively cool air diverted from the compressor is used to pressurize the wheel space and reduce ingress. Also, rim seals have been introduced at the wheel space periphery to find an optimal condition since purge flow interaction with mainstream flow in the annulus can lead to reduced stage performance in terms of efficiency, as has been investigated by (Dahlqvist, 2017; Dahlqvist and Fridh, 2017). Scobie et al. (2016) reviewed the ingress phenomenon from various test rigs and an orifice model was established to relate the seal effectiveness to the purge flow. Shear and the interaction between stationary and rotating components can lead to the development of large scale unsteady flow structures (Beard et al., 2017). These instabilities can be rotationally driven and associated with the disc pumping effect, or pressure driven and associated with pressure asymmetries in the annular gas path with vanes and blades on the stator and rotor respectively (Camci et al., 2019). Studies have been conducted to estimate the rotational instability frequency and structure, in order to 
physically link these structures to their causal phenomenon. Two of the popular postulated theories are that Taylor-Couette and/or Kelvin-Helmhotz phenomena are the driving mechanisms for these instabilities (Horwood et al., 2019). Horwood et al. conducted their study in collaboration with KTH using a replica of the cavity geometry used in the current study. Recently, a different mechanism involving the development of inertial waves was identified as more probable cause of instability (Gao et al., 2020). The number of instabilities and their rotational frequency relative to the disk has been shown to be a function of the purge flow rate. These instabilities exist in cases under rotationally-induced ingress with no vanes or blades, and also under externally-induced ingress with vanes and blades present as has been shown in Savov et al., 2016.

The present work deals with correlating and comparing the behavior of the seal effectiveness with a collaborating university using a geometrically identical test rig but with operating conditions over a wide range of rotational Reynolds numbers and Mach numbers. Here the effect of flow coefficient $\left(C_{F}\right)$ on ingress is investigated. Additionally, unsteady pressure measurements have been conducted to assess the influence of $C_{F}$ on the rotational structures discussed above. The investigation includes a study of the pitch-wise variation in order to examine the growth and decay in amplitudes of these rotational structures.

\section{Methodology}

The experimental facility that has been used is the cold flow Test Turbine Rig at the division of Heat and Power Technology, KTH. The single-stage setup consists of 42 stacked prismatic stator vanes with a positive lean, 60 rotor blades of high pressure gas turbine stage design, and a chute seal at the periphery of the wheel-space between the rotor and the stator. The turbine receives the inlet flow from a screw compressor achieving a maximum pressure of $4 \mathrm{bar}(\mathrm{a})$ at a nominal annular mass-flow rate of $4.7 \mathrm{~kg} / \mathrm{s}$. Figure 1 is a sectional view of the stage geometry illustrating the essential measurement points. The arrows indicate the annular (larger arrow) and purge flow (smaller arrow) inlets and flow direction.

Steady measurements of effectiveness are made using static pressure taps on the stator wall within the cavity.

- The measurement location $5 \mathrm{f}$ corresponds to the lowest radial measurement near the purge inlet at $55 \%$ hub radius.

- The measurement location $5 \mathrm{~d}, 5 \mathrm{~g}, 5 \mathrm{~h}, 5 \mathrm{i}$ correspond to $86 \%, 78 \%, 73 \%$, and $61 \%$ of the hub radius respectively.

- The measurement location $5 \mathrm{~b}$ is the highest position for measurement of static pressures within the cavity at $98.3 \%$ of the hub radius. At this position there are five pitch-wise positions relative to the vanes corresponding to one stator vane pitch of $8.57^{\circ}$. These are shown in the circumferential view in Figure 1.

Unsteady measurements are made using DTS high acquisition system with XCS-062 Kulite pressure transducers, which were set at a sampling frequency of $500 \mathrm{kHz}$. The data was recorded in voltage and translated to pressure using sensor calibration coefficients. The pressure transducers are flush mounted and were not subject to a dynamic calibration. The bandwidth chosen before filtering the data was less than $250 \mathrm{kHz}$, in order to avoid inaccuracies from aliasing. The Kulite sensors had natural frequencies at approximately $275 \mathrm{kHz}$, which also lay outside the zone of interest for all the unsteady analysis.

- The measurement location $5 \mathrm{c}$ is at $94.2 \%$ of hub radius, within the cavity on the stator side. This position also has four pitch-wise positions: three are at circumferential intervals of $15^{\circ}$ between 85 and $115^{\circ}$; the fourth transducer is located at $180^{\circ}$ shift from the one at $100^{\circ}$.

- The measurement location $5 \mathrm{e}$ is the lower cavity position at $75 \%$ of hub radius.

- Positions 5a and 6a correspond to unsteady measurements locations near the casing.

Table 1 shows the operating conditions for the experiments. The purge flow was introduced into the wheelspace from a separate air supply system controlled via three Bronkhorst mass-flow meters. The density ratio (between annular main flow at section 5 and cavity purge flow) varied between 0.985 and 0.998 , assuming ideal gas behaviour in these operational conditions. For the effectiveness measurements, $\mathrm{CO}_{2}$ was seeded into the cavity purge such that it was always at $1 \%$ mass fraction of the total purge mass flow. The static pressure taps were used to extract the $\mathrm{CO}_{2}$ concentration on the stator using a Rosemound NGA Gas Analyzer. 


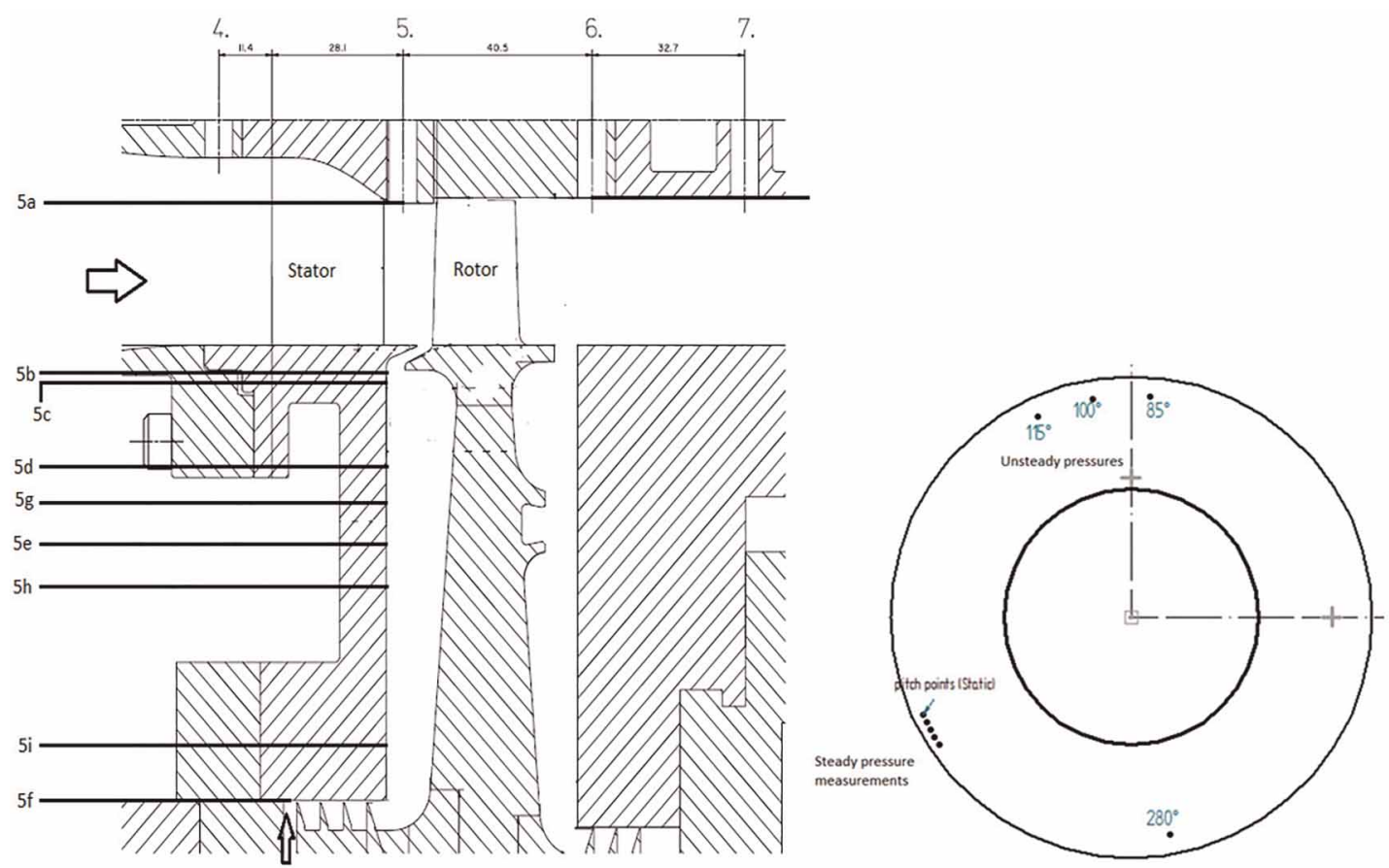

Figure 1. Sectional view of test rig and measurement locations.

In this study the purge flow $\left(\Phi_{0}\right)$ is normalized for calculations as shown below. Note that all symbols are defined in the nomenclature.

$$
\begin{gathered}
\Phi_{0}=\frac{U_{L}}{(\Omega b)} \\
U_{L}=\frac{\dot{m}_{L}}{(2 \pi \rho b s)}
\end{gathered}
$$

The concentration of $\mathrm{CO}_{2}$ was measured by the NDIR (non-dispersive Infra-Red) method, which involves the measurement of concentration by the absorption of infra-red energy by the gas molecules. The concentration effectiveness is defined as follows:

$$
\varepsilon=\frac{\left(c-c_{a}\right)}{\left(c_{0}-c_{a}\right)}
$$

Here the purge inlet concentration $\left(c_{a}\right)$ is based on measurements conducted at $5 \mathrm{f}$ in Figure 1, which is immediately upstream of the labyrinth seal at the inlet of the wheel-space cavity. Further concentration measurements were obtained over a range of static-pressure taps to determine the radial distribution of effectiveness. The uncertainty for concentration measurements is discussed in the Appendix.

The operating points specified below are achieved at a stage pressure ratio (static - static denoted as П) of 2.12 across the sections 4 and section 7 shown in Figure 1. The flow coefficient has been changed by altering the rotational speed of the turbine. The change in swallowing capacity of the turbine at different flow coefficients resulted in small changes in the mass-flow rate, but this was maintained at close to $3.57 \mathrm{~kg} / \mathrm{s}$. With the change in flow coefficient and flow capacity there is a tendency for the pressure ratio to change, this was avoided by careful control of a downstream exhaust fan and upstream valves to ensure fixed pressures at section 4 and 7 for defining the correct operating points.

The effectiveness is directly related to metal temperature, and hence stress and operating life. Here effectiveness was measured from concentration measurements, which are not available in the engine. Appropriate purge flow rates have been determined to seal the wheel-space. From a practical perspective, the engine designer must translate pressure differences between the rim seal and wheel-space entrance to an equivalent effectiveness over a range of purge. 
Table 1. Operating conditions.

\begin{tabular}{|l|l|l|}
\hline $\begin{array}{l}\text { Flow coefficient } \\
\left(C_{F}\right)\end{array}$ & $\begin{array}{l}\text { Rotational Reynolds } \\
\text { Number }\left(\mathrm{Re}_{\boldsymbol{\phi}}\right)\end{array}$ & $\begin{array}{l}\text { Mach number downstream of stator vane exit (Location: } \\
\text { Section 5) (Absolute reference frame) }\end{array}$ \\
\hline 0.83 & $1.45 \times 10^{6}$ & 0.71 \\
\hline 0.585 & $2.11 \times 10^{6}$ & 0.73 \\
\hline 0.52 & $2.39 \times 10^{6}$ & 0.73 \\
\hline 0.45 & $2.74 \times 10^{6}$ & 0.73 \\
\hline 0.41 & $2.98 \times 10^{6}$ & 0.72 \\
\hline 0.35 & $3.54 \times 10^{6}$ & 0.72 \\
\hline 0.325 & $3.7 \times 10^{6}$ & 0.72 \\
\hline 0.3 & $4.14 \times 10^{6}$ & 0.72 \\
\hline
\end{tabular}

A consequence of the methodology is that $C_{F}$ (which is defined in Equation 4 shown below) could not be completely independently controlled in terms of Mach number $(M)$ and Rotational Reynolds number $\left(\operatorname{Re}_{\varphi}\right)$, as slight variations have been shown in Table 1 for the Mach number in absolute frame of reference.

$$
C_{F}=\frac{c_{a x}}{u} \text { and } u=\Omega b
$$

Below it is shown that the effectiveness measured in the wheel-space and the number of rotating instabilities is broadly independent of $M$ and $\operatorname{Re}_{\varphi}$. Note that the independence of effectiveness with $\operatorname{Re}_{\varphi}$ has been well documented - see (Scobie et al., 2016) and (Horwood et al., 2019). The definition of rotational Reynolds number is:

$$
\operatorname{Re}_{\phi}=\rho \Omega b^{2} / \mu
$$

\section{Results and discussion}

\section{Steady results}

This section discusses steady measurements, assessing the degree of ingress in terms of concentration effectiveness $(\varepsilon)$ defined by Equation 3 - see equations below the nomenclature. For the given vane and blade, and chute-seal geometries tested here, effectiveness is investigated in terms of the following non-dimensional parameters: nondimensional purge flow $\left(\Phi_{0}\right)$, radial position on the stator $(r / b)$, circumferential position relative to the vane $(\theta)$, and flow coefficient $\left(C_{F}\right)$. All parameters are defined in the nomenclature.

The variation of concentration effectiveness with flow coefficient for the chute seal is shown in the Figure 2. Here the non-dimension purge flow was set at $\Phi_{0}=0.05$. Also shown in the in the Figure is the deviation of relative flow angle at the rotor blade inlet, $\Delta \beta$. This provides a physical representation of the change in flow coefficient, where the deviation is calculated from the design operating point: $\Delta \beta=0$ at $C_{F}=0.35$.

As would be expected, the degree of ingress is higher at larger radius $(r / b=0.984)$. Effectiveness is larger at $86 \%$ hub radius. At high radius the effectiveness of the chute decreases monotonically with increasing $C_{F}$. There are differences in effectiveness depending on the pitch-wise positions, revealing an asymmetry related to the relative position of the vane.

At the lower radial position, $r / b=0.86$, Figure 2 reveals a minimum at $C_{F}=0.45$, followed by a small increase in effectiveness with increasing CF. Note however that for $C_{F}>0.45, \Delta \beta>35^{\circ}$ and the stage is operating at conditions far from the design point. Figure 3 shows the effect of increased purge at $r / b=0.86$. As expected, the 
effectiveness increases with increasing purge as the wheel-space is pressurized and ingress is reduced. Also the magnitude of the variation of effectiveness with $C_{F}$ decreases as ingress is suppressed. The value of $C_{F}$ for the minima of effectiveness increases with increasing purge as has been evident in Figure 3. High effectiveness is indicative of a reduction of ingress due to presence of the purge flow pushing out instabilities from the chute seal and reducing the pressure deficit within the cavity.

Figure 3 illustrates a slight increase in ineffectiveness at the $86 \%$ radial position for $C_{F}>0.45$. For $C_{F}<0.45$ the trend is reversed. A similar trend was observed by (Savov and Atkins, 2017) for higher flow coefficients between $79 \%$ and $93 \%$ radius irrespective of the vane exit Mach number.

This increase was linked to the reduction of dominant rotational effects with reduced disk rotational speeds, which in turn reduced mixing near the seal and thereby reduced ingress transport deep into the cavity. This would ensure that deeper portions of the cavity might recover some effectiveness due to radial penetration of ingestion with a constant purge flow.

Figure 4 shows the variation of pressure coefficient within the cavity with $C_{F}$, see Equation (6)

$$
C_{P}=\frac{\left(p-p_{\mathrm{ref}}\right)}{\left(0.5 \rho \Omega^{2} b^{2}\right)}
$$
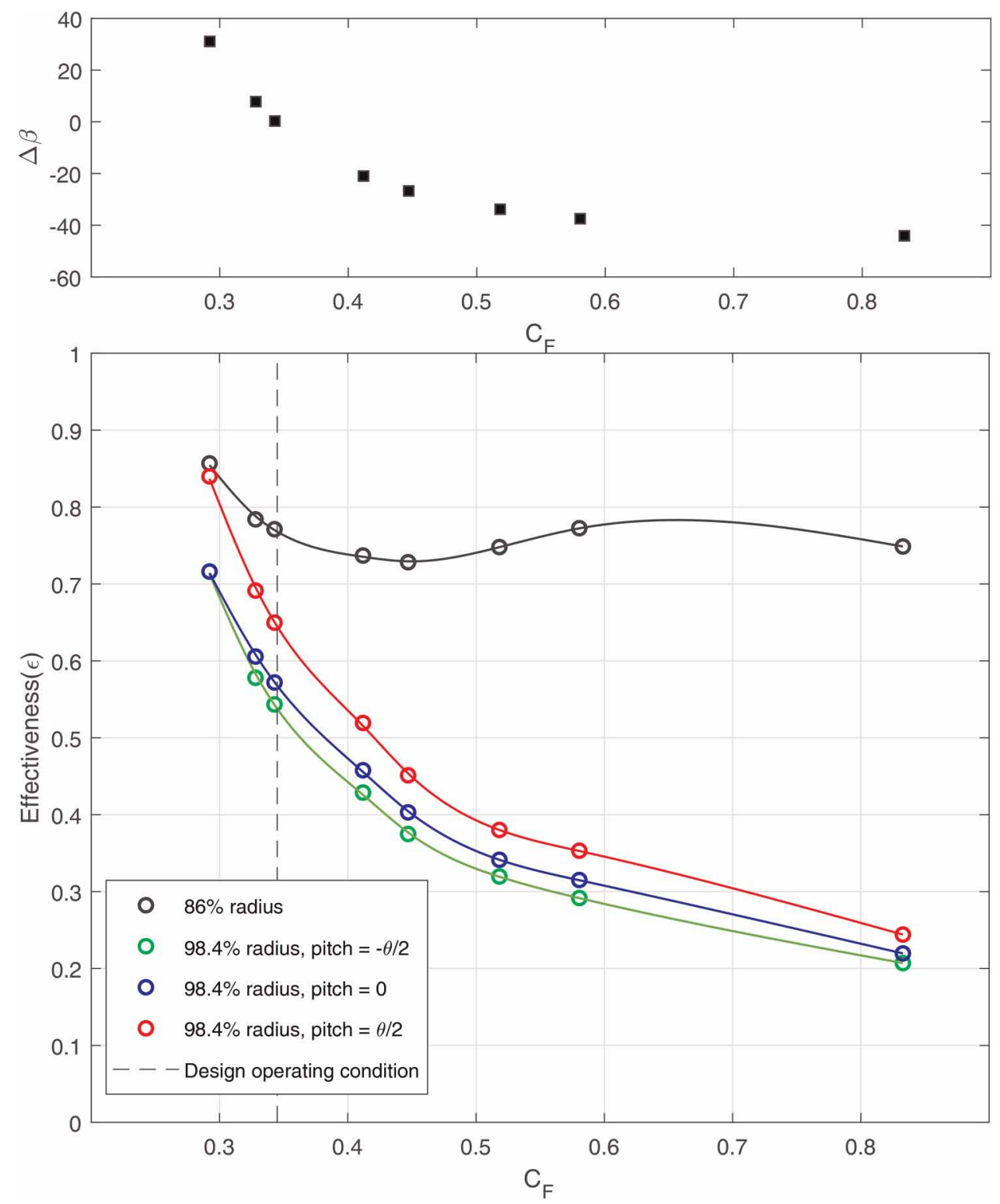

Figure 2. Variation of seal effectiveness with changing flow coefficients at a normalized purge flow rate of $\Phi_{0}=$ 0.05 . 


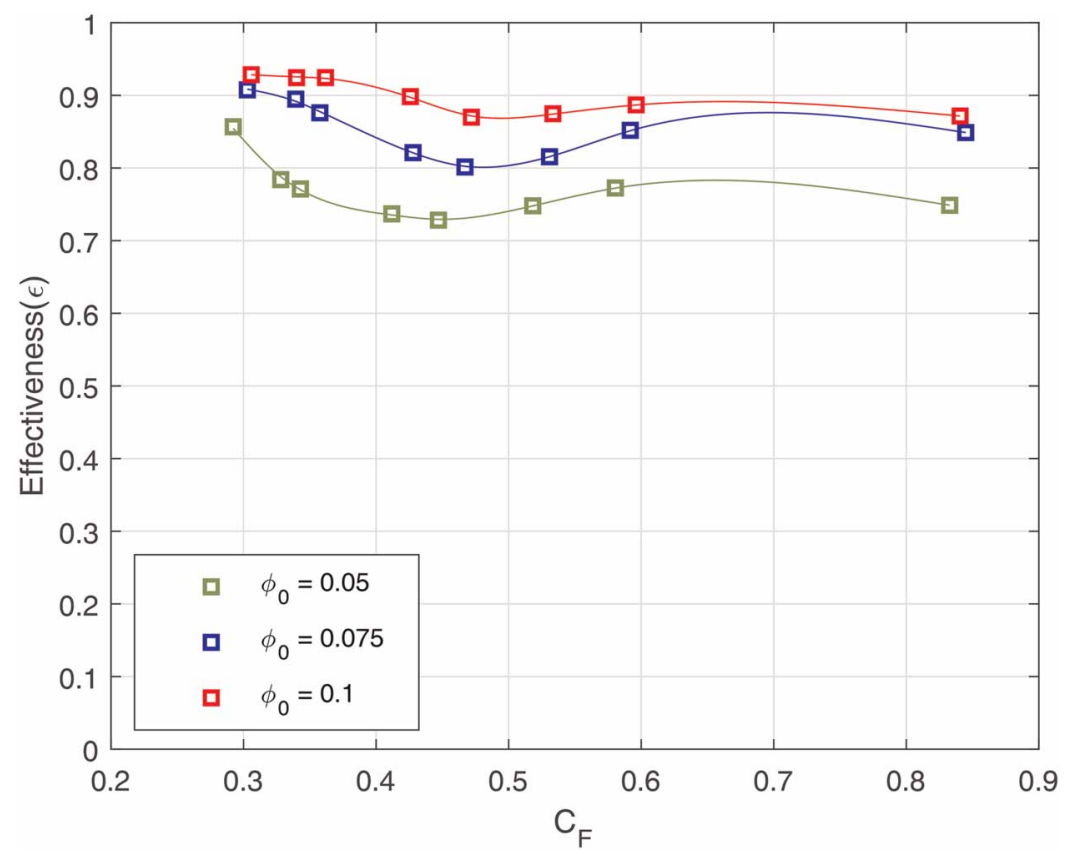

Figure 3. Effectiveness variation with $C_{F}$ at different purge flow rates at $86 \%$ of hub radius.

With a decrease in flow coefficient there is a reduction of pressure coefficient gradient with the radius, which is an indication of a pressure deficit within the cavity. A similar trend was observed by Horwood et al. (2019) with varying purge flow rates, where higher purge flow rates had similar impact on the pressure distribution

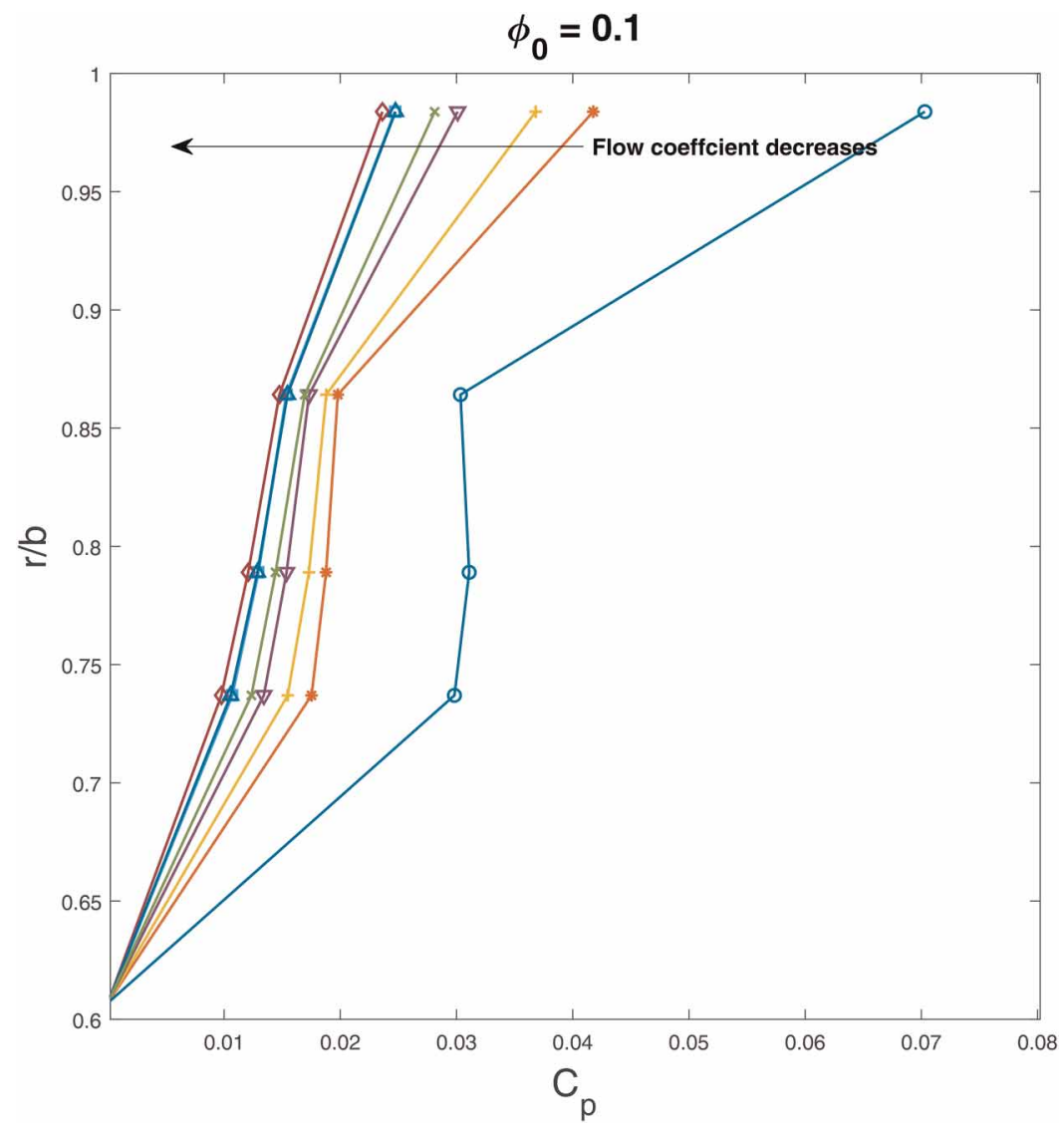

Figure 4. Radial variation of pressure coefficient with flow coefficient for a constant normalized purge flow of $\Phi_{0}=$ 0.1 , inside the cavity. 
within the cavity. Consequently, at lower flow coefficients the effectiveness increases as it would while increasing the purge flow. This behavior can be attributed to the reduction of ingress, especially the externally induced part which involves stator wake and rotor upstream pressure field effects.

The pressure distribution in the annulus is changing with $C_{F}$ as will the interaction of the mainstream and egress with the rotor blades as $\Delta \beta$ changes. Both $\mathrm{KTH}$ and Bath have shown $\Delta C_{p}^{1 / 2}$ on the annulus hub increases linearly with $C_{F}$. There can be an expected increase in ingress as $C_{F}$ increases owing to the greater peak to trough pressure variation across a stator pitch. This will contribute to increased ingress into the cavity and lower the effectiveness as has also been observed by (Dahlqvist, 2017). Furthermore, the increase in flow coefficient means reduction of disk rotational speed, which in turn reduces the speed of the flow thorough the chute seal cavity. So for a relatively constant stator exit flow velocity (which is ensured thorough a constant pressure ratio), the difference in resultant tangential velocity increases (Savov and Atkins, 2017). This increased activity within the seal would lead to lower seal effectiveness for a relatively constant egress rate and hence contribute to increased ingress along the stator wall.

The gap in these plots for $0.59<C_{F}<0.85$ is because operating points were restricted to avoid large mechanical vibrations.

Figure 5 also depicts the variation of effectiveness with radius (normalized), the effect of pushing instabilities out of the cavity and reducing pressure deficit is evident as the effectiveness increases with purge flow. But the gradient of effectiveness change is much higher for radius greater than $85 \%$ in comparison to between $60 \%$ and $80 \%$ radius. This trend is very similar to findings from (Horwood et al., 2019; Robak et al., 2019), which is attributed to the high possibility of impingement of the rotor boundary layer on the stator wall for a chute seal. A consequence of this would be a larger mixing region with interaction between the ingested flow and purge flow pumped thorough the rotor boundary layer.

The Figure 6 shows the variation of effectiveness with non-dimensional purge flow rate at two radial locations. Here two different pressure ratios were used to create two stator exit Mach numbers at a constant flow coefficient, $C_{F}=0.35$. At low radius, effectiveness is seen to increase monotonically with purge. At high radius there is an inflection in the curve, similar to that observed by (Horwood et al., 2019). The sealing effectiveness is usually slightly lower for the higher Mach (higher $\operatorname{Re}_{\phi}$ ) case as had been observed in (Savov and Atkins, 2017).

This was attributed to the possible reduced viscous damping at higher $\operatorname{Re}_{\phi}$ resulting in more mixing. However, the difference in the effectiveness is generally within the measurement uncertainty, except for very low normalized purge flows of below 0.025 . Thus, for this specific flow coefficient both Mach number and $\mathrm{Re}_{\phi}$ independence (weak sensitivity of effectiveness) is observed.

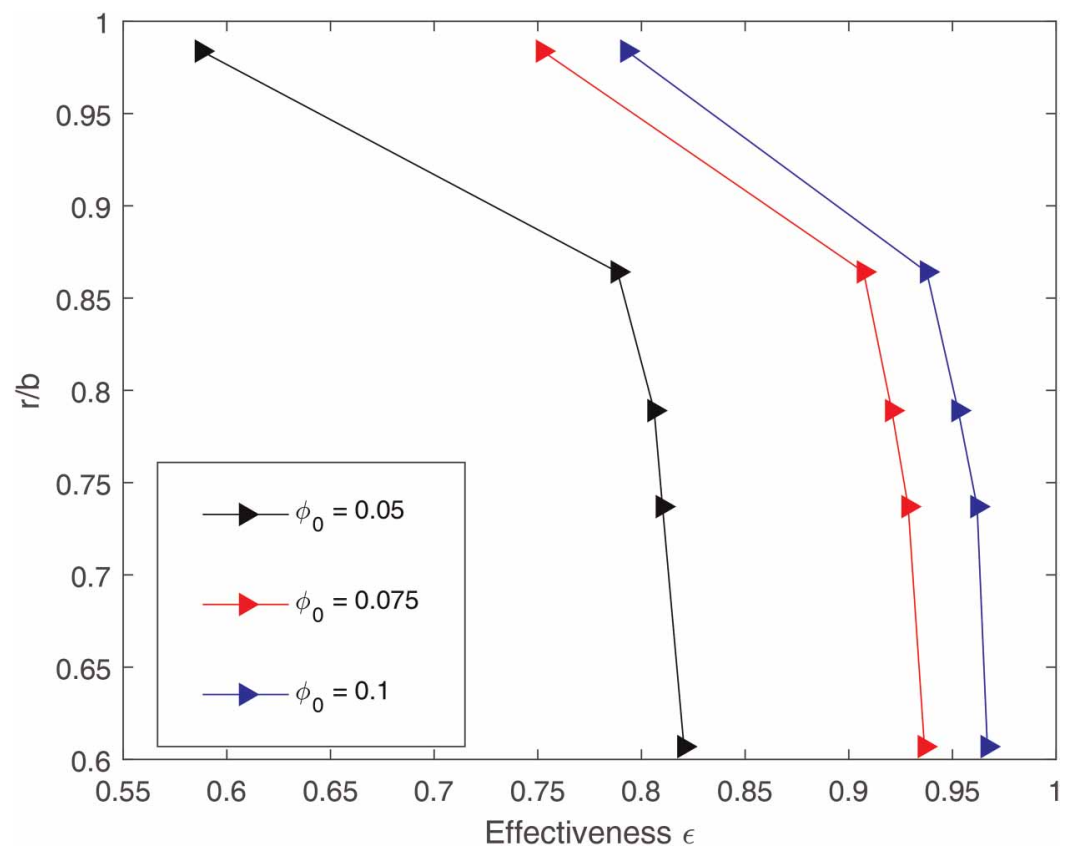

Figure 5. Effectiveness variation with radius, at different purge flow rates at a specific $C_{F}=0.35$. 


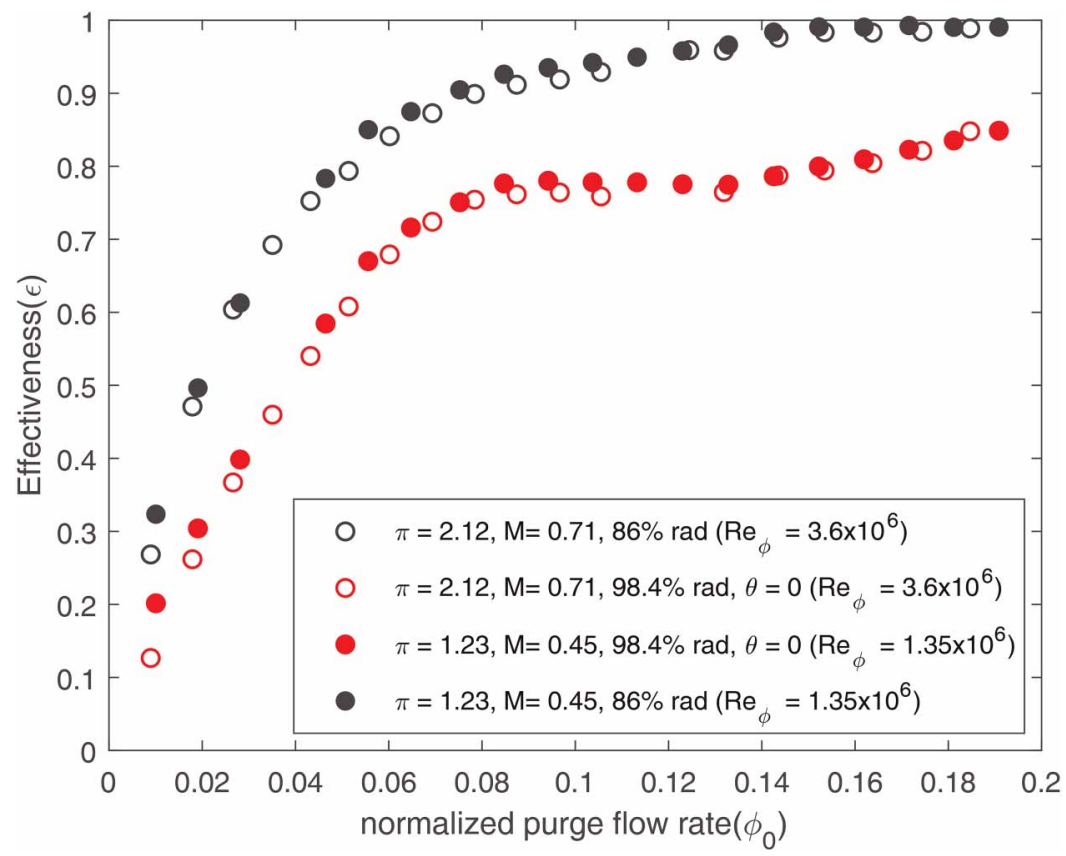

Figure 6. Variation of effectiveness with purge flow for $C_{F}=0.35$ at different $\operatorname{Re}_{\phi}$ and vane exit Mach number.

\section{Unsteady results}

The unsteady experiments were conducted at operational conditions identical to the steady tests. The sampling frequency was set at $500 \mathrm{kHz}$. The unsteady data was pre-processed before conducting Fourier transforms. They were filtered using an FIR low-pass filter of $200 \mathrm{kHz}$ inside the cavity. The sampling rate was checked by resampling the signal to ensure that it is accurate enough to capture the behavior of the disturbances. The truncation of the signal was also controlled to avoid the introduction of divergence by cutting off random time instances. Hence, depending on the disk rotational frequency the signal is truncated at exactly the maximum number of time periods that can be fit into $13 \mathrm{~s}$ of recording time.

The primary region of interest are the frequencies below the blade passing frequency (BPF), which corresponds to 60 (number of rotor blades). These are the frequencies that have been identified by (Horwood et al., 2019) and have been correlated to rotational instabilities that occur between 15 to 25 times the disk frequency. These frequencies have also been used to create notch filters, in order to get an accurate correlation between adjacent signals for tracking the rotational structures within the cavity.

Figure 7 depicts the unsteady pressure coefficient computed from the raw pressure data and the normalised frequency $\mathrm{f} / \mathrm{fd}$ with the $\mathrm{x}$-axis limited to the second harmonic of the BPF. Similar plots were generated the purge flow rates of $\Phi_{0}=0.075$ at four different flow coefficients with rotational speeds of 4,500, 7,250,10,700, and $11,500 \mathrm{rpm}$ (corresponding $C_{F}$ values shown in Table 1) to span the operational range of the turbine at equal intervals - however, the gap between 7,250 and 10,700 was intentional to remove any structural interactions, especially around 9,000 rpm, since the 1 st bending mode of the blades occur at 18 nodal diameters.

Note the data in Figure 7 suggests that there are large-scale structures present between $f \mid f_{d} \sim 18$ and 26 , and that these are unsteady, and possibly changing in count number $(\mathrm{N})$ with time. A similar phenomenon was also reported in (Horwood et al., 2019). There are slight variations that can lead to slight shift in frequencies as they are recorded over $13 \mathrm{~s}$; for the design operating point this comprises of approximately 2,270 rotor revolutions. This is one of the reasons why multiple peaks have been recorded within the aforementioned range of interest.

In Figures 7, 8 and 9, $C_{P}$ is the unsteady pressure magnitude normalized as shown in Equation (7):

$$
C_{P}=\frac{\left(A_{p}\right)}{\left(0.5 \rho \Omega^{2} b^{2}\right)}
$$

In all the FFTs the BPF is the most dominant response, however the disturbances below the BPF have been quite consistent with the range of interest of $18-26 f f f_{d}$. There is evidence of reduction of the magnitude (intensity) of the disturbance peaks due to introduction of higher purge flow from $\Phi_{0}=0.05$ to $\Phi_{0}=0.075$, through the cavity at the design flow coefficient of 0.35. A similar phenomenon was observed by (Horwood et al., 2019). 


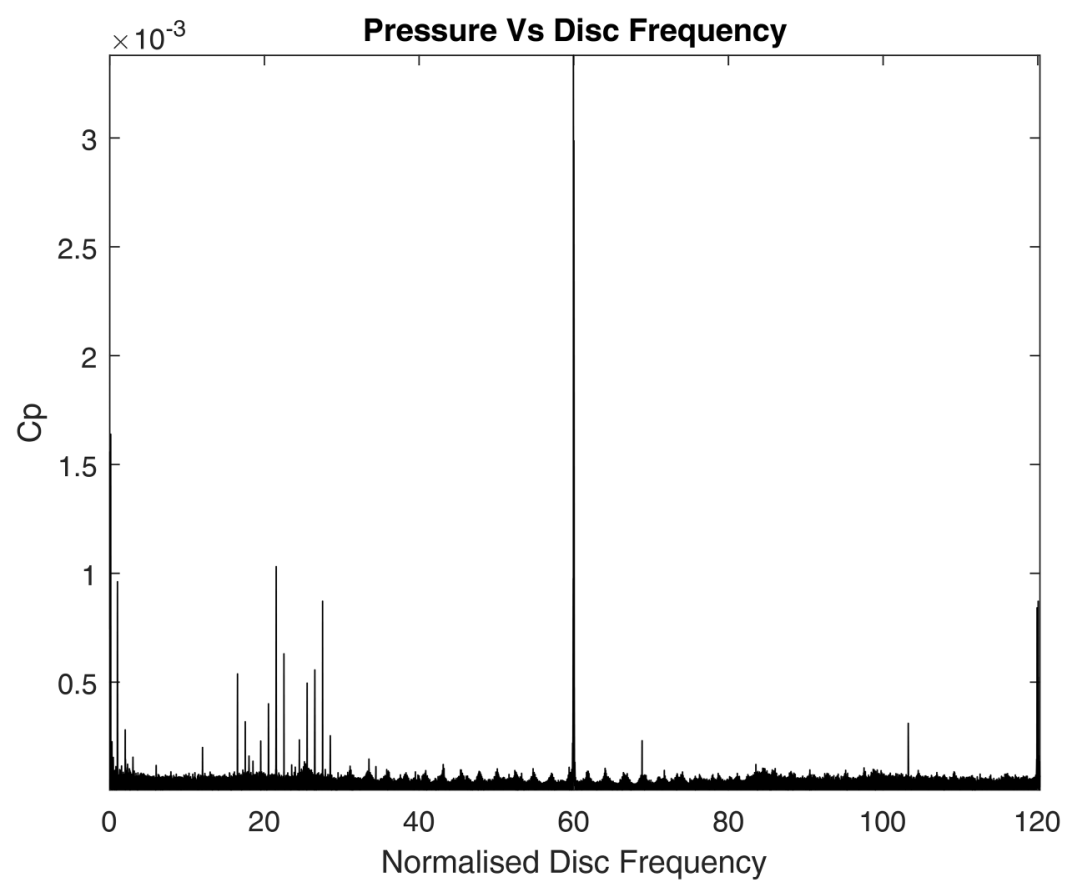

Figure 7. Fast Fourier Transform at $94 \%$ hub radius and pitch of $280^{\circ}$ at $C_{F}=0.35, R e_{\phi}=3.54 \times 10^{6}, \boldsymbol{\Phi}_{0}=0.05$.

Having conducted similar analysis for all the pitchwise measurement locations, perturbations 15-25 and 27.5-30 times the disk rotational frequency revealed periodic growth and decay of amplitude every $15^{\circ}$ between the pitchwise distributed transducers.

Additionally there are disturbances in the lower frequency range of $1-8 f f f_{d}$ and higher range of $110-115 f f_{d}$. These can be witnessed as more pronounced in Figure 9, but these disturbances although are prominent change in frequency with change in rotational speed, which could be indicative of physical interference from local or surface imperfections rather than fluid structures. The disturbances between the range of 18-26 also showed periodic growth and decay especially for the transducer pitch positions of $85^{\circ}, 100^{\circ}$ and $115^{\circ}$, which can be seen while comparing the FFTs from Figures 7 and 9.

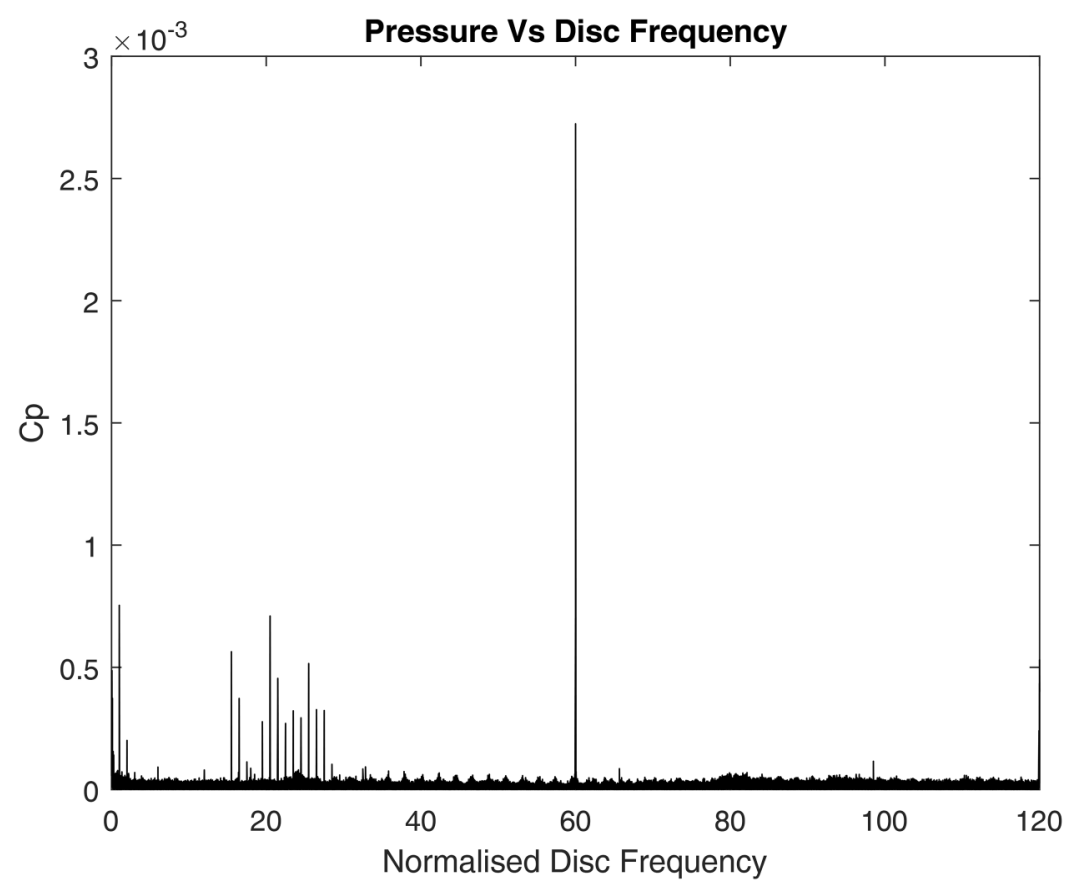

Figure 8. Fast Fourier Transform at $94 \%$ hub radius and pitch of $280^{\circ}$ at $C_{F}=0.35, R e_{\phi}=3.54 \times 10^{6}, \boldsymbol{\Phi}_{0}=0.075$. 


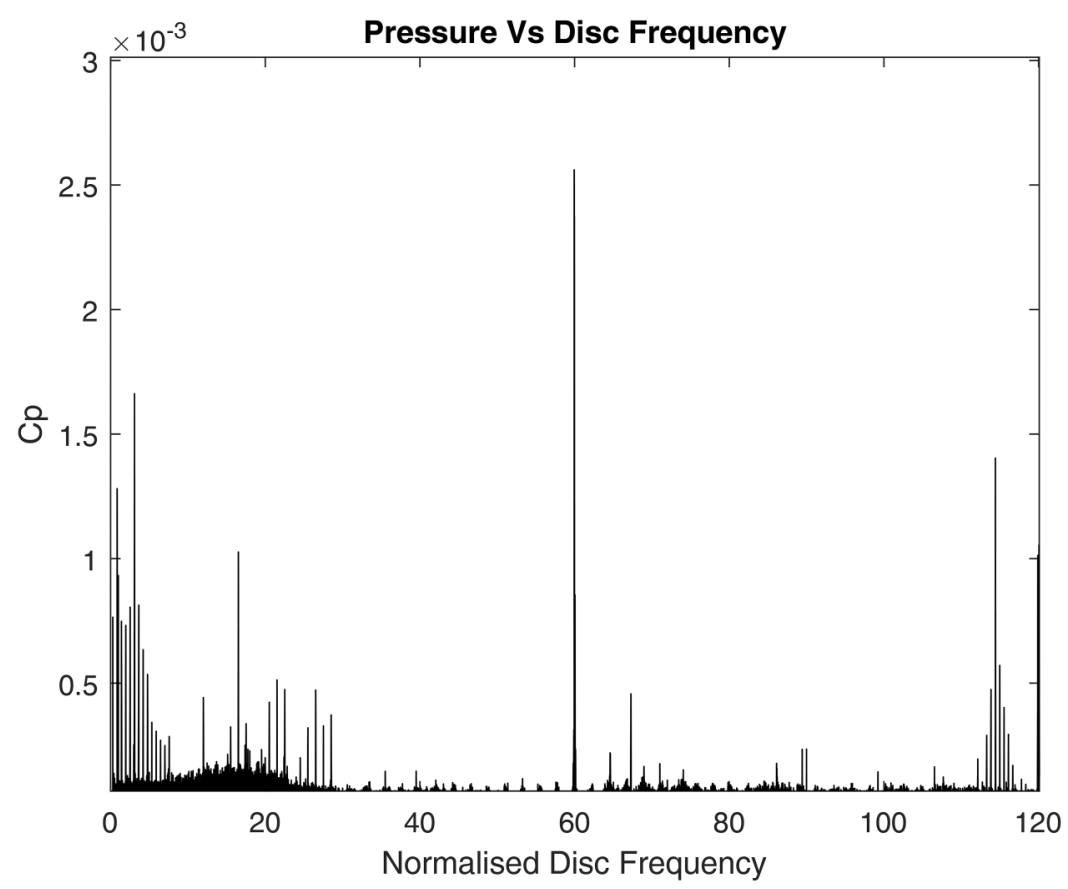

Figure 9. Fast Fourier Transform at $94 \%$ hub radius and pitch of $100^{\circ}$ at $C_{F}=0.35, R e_{\phi}=3.54 \times 10^{6}, \boldsymbol{\Phi}_{0}=0.05$.

However, at the $280^{\circ}$ position shown in these figures the disturbances decay in strength particularly the lower range disturbances between $0.5-10 f f_{d}$ and the higher range disturbances (close to second harmonic of BPF) are almost completely damped, which suggest that if there are rotational structures moving at these frequencies, they are inconsistent and discontinuous as they get dissipated while travelling along the circumference of the wheelspace cavity near the chute seal. The disturbances close to the second harmonic of BPF could be a possible resultant of harmonics and non-linear combinations of the low frequency disturbances. But similar to the lower frequencies this is also inconsistent over the pitch change of $180^{\circ}$ and hence they have not been attributed to any specific consistent rotational structures during the correlation process.

The unsteady flow structures close to the chute seal have been further investigated using cross-correlation between the circumferentially placed sensors at $85^{\circ}, 100^{\circ}, 115^{\circ}$, and $280^{\circ}$. This cross correlation is based on the phase difference between signals as explained by (Beard et al., 2017). A histogram was created using a crosscorrelation algorithm for every rotor time period of revolution and for 800 revolutions. In each revolution the maximum correlation value and the associated phase was extracted. The lag time between the two signals was computed from this information along with the sampling frequency of the sensor, which was $500 \mathrm{kHz}$.

The histogram shown in Figure 10 was utilized to estimate the number of peaks found within each interval of normalized lag time.

The lag time was used to calculate the rotational speed of the rotating structures, following the method of Beard et al. These cross correlations were primarily conducted using a notch filter, where the frequency of interest was specified and a bandwidth of one disk rotational frequency provided a more accurate correlation. Eventually a scatter plot was created (see Figure 11) where the normalized lag time is distributed in intervals based on the frequency, or frequency range, of interest. Hence the maximum lag time is always computed for every revolution from the histogram and this lag time is normalized by the disk rotational frequency. Subsequently, this operation is repeated for all the possible pairs of the circumferential pitch positions. The vertical axis of Figure 11 represents the angle between these possible pairs of measurement points, and the normalized lag time is distributed on the horizontal axis. The diameter of the scatter points are an approximate representation of the amount of correlation with that specific time lag, i.e. the number of samples (or peaks) passing between those sensors with the calculated lag time.

Following Beard et al., the slope of the dotted lines joining the correlated values between the pitch-wise sensor pairs is used to calculate the rotational speed of the structures. The number of structures is computed by the vertical distance between the intersections of these lines with the correlation values.

The correlation method based on the scatter plots were conducted for four different flow coefficients and for the two normalized flow rates of $\Phi_{0}=0.075$ and 0.05 , respectively. The outcome from the analysis is provided 


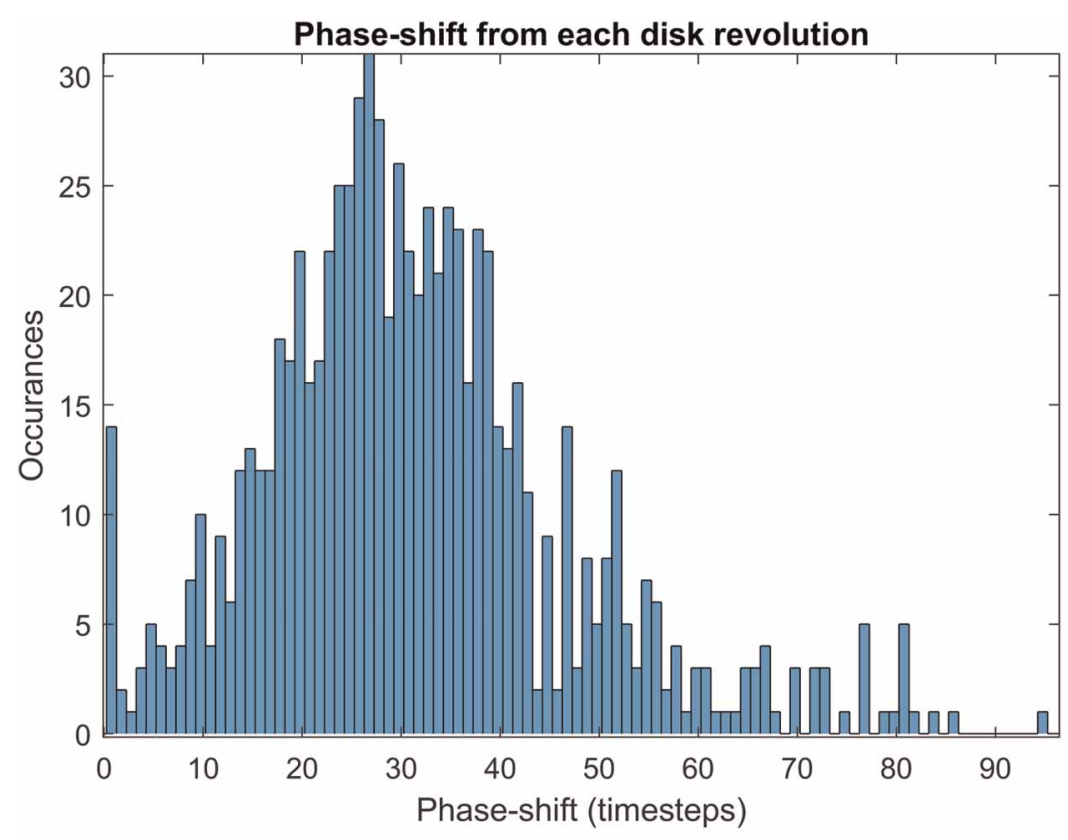

Note:4.132323e+03 timesteps per revolution

Figure 10. Histogram of number of samples associated with the phase shift between the two signals analysed over 800 rotor revolutions at $C_{F}=0.35, \operatorname{Re}_{\phi}=3.54 \times 10^{6}, \boldsymbol{\Phi}_{0}=0.05$.

in Table 2. The results are in accordance with previous published data (Beard et al., 2017; Horwood et al., 2019). The number of structures and their rotational speed are broadly similar to the published data.

Large scale flow structures were reported by (Horwood et al., 2019) for purge flow rates of 0.05 and 0.075 at $C_{F}=0.35$ and $\operatorname{Re}_{\varphi}=1.3 \times 10^{6}$. For disturbances in the range of 18-22 times disk frequency, 21 rotational structures were reported to be moving at $103 \%$ and $92 \%$ of the disk rotational speed. Also, an increase the purge flow rate causes a reduction of frequency and rotational speed of the instabilities identified below the BPF. This is consistent with the data collected here. Additionally, the number of rotational structures also reduces, although by a small margin as observed by Beard et al. Thus, the reduction of the strength and speed of rotation

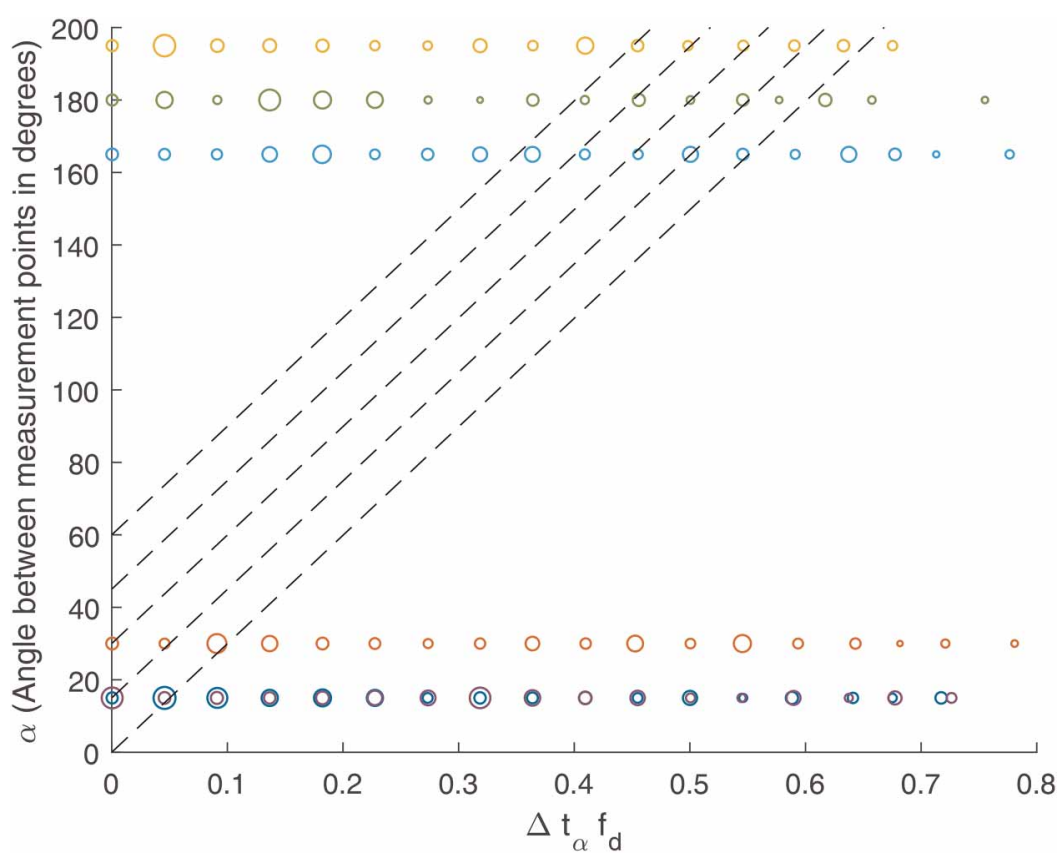

Figure 11. Scatter plot for cross-correlation between all pairs of sensor combinations for normalized purge flow of $\Phi_{0}=0.05$ at $C_{F}=0.35(10,600$ RPM). 
Table 2. Rotational speeds and number of large scale flow structures for various flow coefficients and purge rates.

\begin{tabular}{|c|c|c|c|c|c|}
\hline$\Phi_{0}$ & RPM & $f_{d}(\mathrm{~Hz})$ & $f / f_{d}$ (range) & $\omega / \Omega$ & $N$ \\
\hline \multirow[t]{6}{*}{0.075} & $\begin{array}{l}4,500 \\
C_{F}=0.83\end{array}$ & 75 & $24.5(24-25)$ & 0.92 & 26 \\
\hline & $\begin{array}{l}7,250 \\
C_{F}=0.52\end{array}$ & 121 & $23.3(23-24)$ & 0.92 & 24 \\
\hline & \multirow{2}{*}{$\begin{array}{l}10,700 \\
C_{F}=0.35\end{array}$} & \multirow[t]{2}{*}{178.4} & $20.5(19-20)$ & 0.82 & 24 \\
\hline & & & $25.4(25-26)$ & 0.95 & 26 \\
\hline & \multirow{2}{*}{$\begin{array}{l}11,500 \\
C_{F}=0.32\end{array}$} & \multirow[t]{2}{*}{191.6} & $15.5(15-16)$ & 0.81 & 18 \\
\hline & & & $20.5(20-21)$ & 0.81 & 26 \\
\hline \multirow[t]{6}{*}{0.05} & $\begin{array}{l}4,500 \\
C_{F}=0.83\end{array}$ & 75 & $25(25-26)$ & 0.94 & 27 \\
\hline & $\begin{array}{l}7,250 \\
C_{F}=0.52\end{array}$ & 121 & $24.8(24-25)$ & 0.95 & 26 \\
\hline & \multirow{2}{*}{$\begin{array}{l}10,700 \\
C_{F}=0.35\end{array}$} & \multirow[t]{2}{*}{178.4} & $22(22-23)$ & 0.83 & 26 \\
\hline & & & $26.2(26-27)$ & 0.96 & 26 \\
\hline & \multirow{2}{*}{$\begin{array}{l}11,500 \\
C_{F}=0.32\end{array}$} & \multirow[t]{2}{*}{191.6} & $14.9(14-15)$ & 0.82 & 19 \\
\hline & & & $24.3(24-25)$ & 0.88 & 28 \\
\hline
\end{tabular}

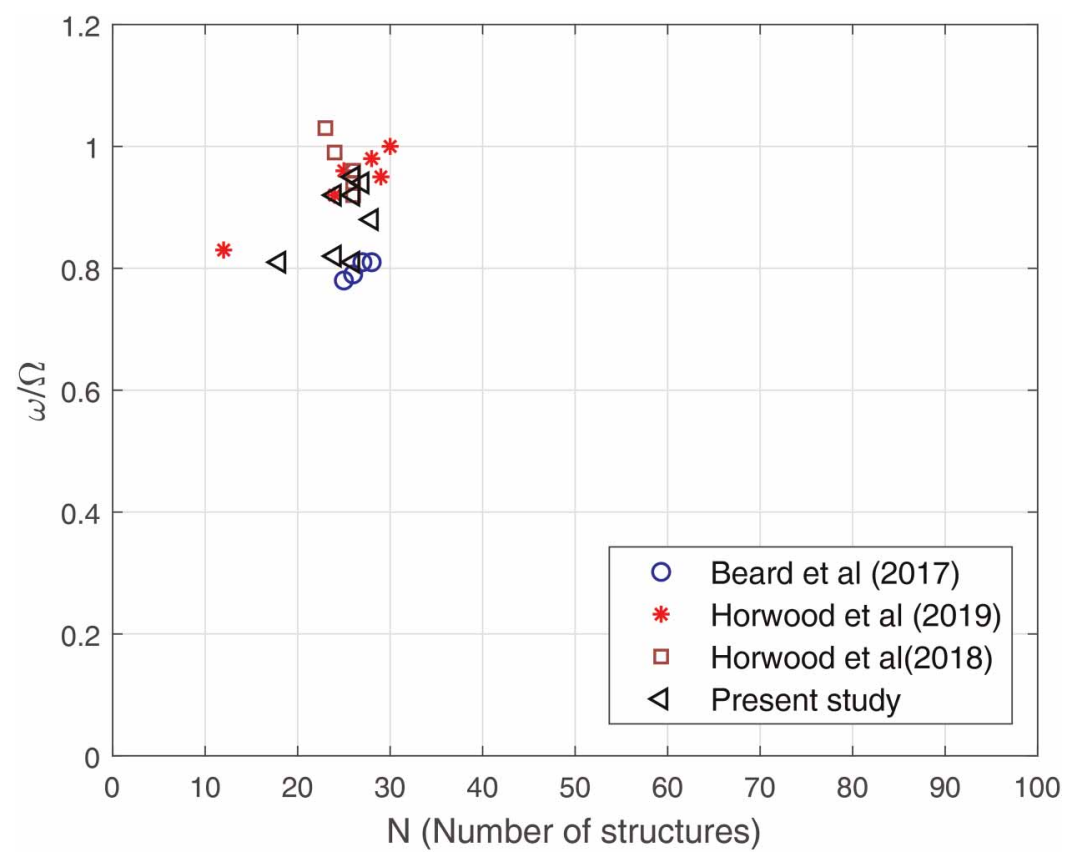

Figure 12. Rotating unsteady pressure structures - comparison with other studies. 
of the large-scale structures is indicative of the stabilization effect caused by increasing the purge. The number of structures and their rotational speed is only weakly influenced by flow coefficient over $0.32<C_{F}<0.85$. Figure 12 compares unsteady data collected here with the published literature; data elsewhere was collected at constant $C_{F}$.

\section{Conclusions}

An experimental investigation has been performed using a single stage turbine with a chute seal at the cavity periphery over a range of flow coefficients and purge flow rates.

- Effectiveness of the chute seal is strongly dependent on purge flow rate and flow coefficient, $C_{F}$. However, at high purge flow rates the sensitivity of effectiveness to $C_{F}$ reduces.

- Effectiveness is weakly influenced by the rotational Reynolds number for a fixed purge flow rate and vane exit Mach number.

- The effectiveness is sensitive to radial positions within the cavity and close to the chute seal the effectiveness is sensitive to the circumferential position relative to the vane.

- The amplitudes and rotational speeds of these disturbances have been observed to be slightly reduced with increased purge flow rates.

The number and rotational speed of the unsteady structures $(N$ and $\omega / \Omega)$ are weakly sensitive to $C_{F}$ and consistent with measurements from other researchers (Beard et al., 2017; Horwood et al., 2019; Horwood et al., 2018; Chew et al., 2019).

Future work should investigate the disturbances for frequencies greater than the BFP, considering the possibility of stator wake vortex shedding. Such phenomena create frequencies identified to exist within the Strouhal number range (with significant intensities/ amplitudes) based on the stator-vane trailing-edge thickness.

\section{Appendix}

The seal effectiveness $(\varepsilon)$ is defined by the equation shown below:

$$
\varepsilon=\frac{c_{s}-c_{a}}{c_{o}-c_{a}}
$$

where the subscripts $a, o$ and $s$ respectively denote the air in the annulus, the sealing air at inlet, and the surface of the stator.

Let $\delta_{\varepsilon}, \delta_{s}, \delta_{0}, \delta_{a}$ be uncertainties in $\varepsilon, c_{s}, c_{0}, c_{a}$ respectively so that

$$
\begin{aligned}
\varepsilon \pm \delta_{\varepsilon} & =\frac{c_{s} \pm \delta_{s}-\left(c_{a} \pm \delta_{a}\right)}{c_{o} \pm \delta_{o}-\left(c_{a} \pm \delta_{a}\right)} \\
& =\frac{\left(c_{s}-c_{a}\right)\left[1+\left( \pm \delta_{s} \pm \delta_{a}\right) /\left(c_{s}-c_{a}\right)\right]}{\left(c_{o}-c_{a}\right)\left[1+\left( \pm \delta_{o} \pm \delta_{a}\right) /\left(c_{o}-c_{a}\right)\right]} \\
& =\varepsilon \frac{\left[1+\left( \pm \delta_{s} \pm \delta_{a}\right) /\left(c_{s}-c_{a}\right)\right]}{\left[1+\left( \pm \delta_{o} \pm \delta_{a}\right) /\left(c_{o}-c_{a}\right)\right]}
\end{aligned}
$$

If $\left( \pm \delta_{0} \pm \delta_{a}\right) /\left(c_{0}-c_{a}\right) \ll 1$ then

$$
\varepsilon+\left|\delta_{\varepsilon}\right| \leq \varepsilon\left\{1+\frac{\left|\delta_{s}\right|+\left|\delta_{a}\right|}{c_{s}-c_{a}}+\frac{\left|\delta_{o}\right|+\left|\delta_{a}\right|}{c_{o}-c_{a}}\right\}
$$

Hence,

$$
\frac{\left|\delta_{\varepsilon}\right|}{\varepsilon} \leq\left\{\frac{\left|\delta_{s}\right|+\left|\delta_{a}\right|}{c_{s}-c_{a}}+\frac{\left|\delta_{o}\right|+\left|\delta_{a}\right|}{c_{o}-c_{a}}\right\}
$$


If the uncertainty is a percentage of the full-scale range, which was the case in the experiments, then $\delta_{s}=\delta_{0}=$ $\delta_{a}=\delta$, say, and Equation (A5) simplifies to

$$
\frac{\left|\delta_{\varepsilon}\right|}{\varepsilon} \leq 2 \delta \frac{1+\varepsilon^{-1}}{c_{o}-c_{a}}
$$

or

$$
\left|\delta_{\varepsilon}\right| \leq 2 \delta \frac{1+\varepsilon}{c_{o}-c_{a}}
$$

The average standard deviation, $\sigma$, in the range $0<\varepsilon<1$ can be calculated from Equation A7 by

$$
\sigma=\frac{2 \delta}{c_{o}-c_{a}} \sqrt{\int_{0}^{1}(1+\varepsilon)^{2} d \varepsilon=3.06 \frac{\delta}{c_{o}-c_{a}}}
$$

The uncertainty analysis has been conducted for the effectiveness measurements. The Rosemound NGA Gas Analyzer used for the $\mathrm{CO}_{2}$ concentration measurements had $1 \%$ of the full scale at a constant pressure and temperature within the measurement range. This would result in a standard deviation of 0.038 .

\section{Nomenclature}

$A_{p} \quad$ Unsteady pressure amplitude $(\mathrm{Pa})$

ax Used in subscript to suggest axial

a Used in subscript is ambient

$b$ disc radius $(\mathrm{m})$

$c_{a}$ Concentration of $\mathrm{CO}_{2}$ at purge inlet

$c_{0}$ Concentration of $\mathrm{CO}_{2}$ at annular inlet

$C_{F} \quad$ Flow coefficient

$C_{P}$ Pressure coefficient

$c_{\mathrm{ax}}$ Axial velocity (annular) $(\mathrm{m} / \mathrm{s})$

$f$ frequency in $\mathrm{Hz}$

$f_{d}$ disk rotational frequency

$\dot{m}_{L} \quad$ Leakage / Purge mass flow rate $(\mathrm{kg} / \mathrm{s})$

$N$ number of rotational structures

$M \quad$ Mach number

$\mathrm{Re}_{\phi}$ rotational Reynolds number

$s$ seal clearance $(\mathrm{m})$

$u \quad$ disk rotational speed $(\mathrm{m} / \mathrm{s})$

$\Phi_{0}$ normalized purge rate

$\omega$ rotational speed of structure $(\mathrm{rad} / \mathrm{s})$

$\Omega$ rotational speed of rotor $(\mathrm{rad} / \mathrm{s})$

$\Pi \quad$ static pressure ratio

$\varepsilon \quad$ seal effectiveness

$\Delta \beta$ elative flow angle deviation at rotor inlet

$\mu \quad$ dynamic viscosity $\left(\mathrm{N} s / \mathrm{m}^{2}\right)$

$\rho$ density $\left(\mathrm{kg} / \mathrm{m}^{3}\right)$

0 purge Inlet

\section{Acknowledgements}

The authors would like to thank Kunyuan Zhou, Gregor Schmidt, Navid Mikaillian for their invaluable inputs and support during the research. Special thanks also to the HPT laboratory engineers Göran Arntyr and Leif Pettersson for their invaluable support with electrical to mechanical issues related to the experiments. 


\section{Funding sources}

The research received funding as part of a collaboration between KTH Royal Institute of Technology and SIEMENS Turbomachinery AB, Finspång.

\section{Competing interests}

Arijit Sinha Roy declares that he has no conflict of interest. Jens Fridh declares that he has no conflict of interest. James A. Scobie declares that he has no conflict of interest. Carl M. Sangan declares that he has no conflict of interest. Gary

D. Lock declares that he has no conflict of interest.

\section{References}

Beard P. F., Gao F., Chana K. S., and Chew J. (2017). Unsteady flow phenomena in turbine rim seals. ASME Journal of Engineering for Gas Turbines and Power. September 27, 2016; 139 (3): 032501. https://doi.org/10.1115/1.4034452.

Camci C., Averbach M., and Town J. (2019). Unsteady flow structures within a turbine rim seal cavity in the presence of purge flow- An experimental and computational unsteady aerodynamics investigation. Aerospace. 6 (5): 60. https://doi.org/10.3390/aerospace6050060.

Chew J. W., Gao F., and Palermo D. M. (2019). Flow mechanisms in axial turbine rim sealing. Journal of Mechanical Engineering Science. 233 (23-24): 7637-7657. https://doi.org/10.1177/0954406218784612.

Dahlqvist J. (2017). Cavity purge flows in high pressure turbines. Stockholm: KTH Royal Institute of Technology.

Dahlqvist J. and Fridh J. (2017). Experimental investigation of turbine stage flow field and performance at varying cavity purge rates and operating speeds. ASME Journal of Turbomachinery. March 2018; 140 (3): 031001. https://doi.org/10.1115/1.4038468.

Gao F., et al. (2020). Inertial waves in turbine rim seal flows. Physical Review Fluids. 5: 13-14. https://doi.org/10.1103/PhysRevFluids.5. 024802 .

Horwood J. T. M., Hualca F. P., Scobie J. A., Wilson M., Sangan C. M., et al. (2018). Experimental and computational investigation of flow instabilities in turbine rim seals. ASME Journal of Engineering for Gas Turbines and Power. January 2019; 141 (1): 011028. https://doi.org/10.1115/1.4041115.

Horwood J. T., Hualca P. F., Wilson M., Scobie J., Sangan C. M., et al. (2019). Flow instabilities in turbine chute seals. ASME Journal of Engineering for Gas Turbines and Power. 141. [GT2019-90982]. https://doi.org/10.1115/1.4045148.

Robak C., Faghri A., and Thole K. (2019). Analysis of Gas Turbine Rim Cavity Ingestion With Axial Purge Flow Injection. https://doi. org/10.1115/GT2019-91807.

Savov S. S., Atkins N. R., and Uchida S. Comparison of single and double lip rim seal geometry. In: Proceedings of the ASME Turbo Expo 2016: Turbomachinery Technical Conference and Exposition. Volume 5A: Heat Transfer, Seoul, South Korea, June 13-17, 2016.

Savov S. S. and Atkins N. R. (2017). A Rim Seal Ingress Model Based on Turbulent Transport. ASME. https://doi.org/10.1115/ GT2017-63531.

Scobie J. A., Sangan C. M., Michael Owen J., and Lock G. D. (2016). Review of ingress in gas turbines. ASME Journal of Engineering for Gas Turbines and Power. 138 (12): 120801. https://doi.org/10.1115/1.4033938. 